\title{
Reference Protocol to Assess Analytical Performance of Higher Order Structural Analysis Measurements: Results from an Interlaboratory Comparison
}

\author{
K. Groves,* A. E. Ashcroft, A. Cryar, A. Sula, B. A. Wallace, B. B. Stocks, C. Burns, D. Cooper-Shepherd, \\ E. De Lorenzi, E. Rodriguez, H. Zhang, J. R. Ault, J. Ferguson, J. J. Phillips, K. Pacholarz, K. Thalassinos, \\ L. Luckau, L. Ashton, O. Durrant, P. Barran, P. Dalby, P. Vicedo, R. Colombo, R. Davis, R. Parakra, \\ R. Upton, S. Hill, V. Wood, Z. Soloviev, and M. Quaglia*
}

Cite This: Anal. Chem. 2021, 93, 9041-9048

Read Online

ACCESS | Llll Metrics \& More | 回 Article Recommendations | sl Supporting Information

ABSTRACT: Measurements of protein higher order structure (HOS) provide important information on stability, potency, efficacy, immunogenicity, and biosimilarity of biopharmaceuticals, with a significant number of techniques and methods available to perform these measurements. The comparison of the analytical performance of HOS methods and the standardization of the results is, however, not a trivial task, due to the lack of reference protocols and reference measurement procedures. Here, we developed a protocol to structurally alter and compare samples of somatropin, a recombinant biotherapeutic, and describe the results obtained by using a number of techniques, methods and in different laboratories. This, with the final aim to provide tools and
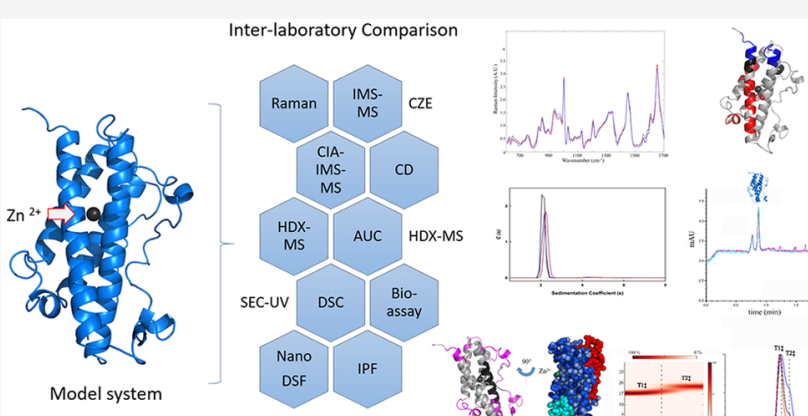
generate a pool of data to compare and benchmark analytical platforms and define method sensitivity to structural changes. Changes in somatropin HOS, induced by the presence of zinc at increasing concentrations, were observed, both globally and at more localized resolution, across many of the methods utilized in this study and with different sensitivities, suggesting the suitability of the protocol to improve understanding of inter- and cross-platform measurement comparability and assess analytical performance as appropriate.
$\mathrm{M}$ easurements of protein higher order structures (HOS) provide information on the stability, potency, efficacy, immunogenicity, and biosimilarity of biotherapeutics and an insight of the dynamics and functionality of proteins. ${ }^{1-4} \mathrm{~A}$ wide variety of techniques are available for the analysis of protein HOS, but little is known about the comparability of the data generated. Here, we developed, for the first time, a protocol to prepare and progressively modify the structure of a protein standard, to assess sensitivity to structural changes of different analytical methodologies and enable measurement comparability studies. This, to our knowledge, is also the first interlaboratory comparison of its kind.

Somatropin (recombinant human growth hormone, rhGH) was chosen as the protein model system in this study as it is a stable, well characterized protein, ${ }^{5-7}$ with a defined HOS. Furthermore, it is easily and reliably sourced. ${ }^{8,9}$ A reference protocol was here developed to progressively induce localized HOS changes via the addition of zinc salts. The effects of $\mathrm{Zn}^{2+}$ on hGH are well documented in the literature. ${ }^{5,10-12} \mathrm{Zn}^{2+}$ is known to induce dimerization of hGH in the pituitary glands, ${ }^{12}$ and its use as a precipitating agent in formulation processing has revealed minor tertiary structural changes of $\mathrm{rhGH}^{13}$
Hydrogen-deuterium exchange-mass spectrometry (HDXMS), ion mobility spectrometry-mass spectrometry (IMS-MS), size exclusion chromatography (SEC) and thermal stability experiments were first carried out to define the experimental conditions to consistently induce rhGH structural changes via $\mathrm{Zn}^{2+}$. An interlaboratory comparison was then organized, where data were acquired by HDX-MS, IMS-MS, Raman spectrometry (Raman), analytical ultracentrifugation (AUC), circular dichroism spectroscopy (CD), capillary zone electrophoresis (CZE), thermal stability, and biological activity measurements, to assess the potential of the protocol to be used to define instrument performance and carry out comparability measurements across platforms.

Received: November 2, 2020

Accepted: June 7, 2021

Published: June 24, 2021 


\section{EXPERIMENTAL SECTION}

Chemicals and Reagents. All chemicals and standards were purchased from Sigma-Aldrich [Gillingham, U.K.] and were of research grade purity unless otherwise stated. Ultrapure water $\left(18 \mathrm{M} \Omega \mathrm{cm}^{-1}\right)$ was used. For buffer and sample preparation, monobasic and dibasic potassium phosphate and HPLC grade water were purchased from Acros Organics [Belgium].

The following materials were supplied to each participating laboratories: ampules each containing $1.9 \mathrm{mg}$ of lyophilized rhGH standard [WHO 98/574, NIBSC, U.K.]; vials containing, respectively, $0.165 \mathrm{~g}$ of zinc acetate, $0.18 \mathrm{~g}$ of potassium phosphate monobasic, and $0.64 \mathrm{~g}$ of potassium phosphate dibasic; $1 \mathrm{~L}$ of HPLC grade water; one protein low binding Eppendorf [Eppendorf, U.K.]. All samples and reagents were shipped at room temperature with details on the handling of the samples, sample preparation, and storage as described in the Supporting Information.

Participating Laboratories and Analytical Methods. Thirteen laboratories participated in the interlaboratory comparison, with some laboratories providing multiple data sets from different analytical platforms. Participants were supplied with one of three working instructions (WI), for generic, HDX-MS, and IMS-MS analytical based platforms (Supporting Information), with a detailed description of protein sample preparation, some desired analytical conditions, and a report formatted to feedback methods and results. Participants were assigned a random and anonymized number, from 1 to 13 .

Overall the analysis of rhGH control, rhGH:Zn $1: 2$ and rhGH:Zn 1:10 was performed using 10 techniques: analytical ultracentrifugation, (AUC, Beckman Coulter proteomeLab XL-I), laboratory 9; capillary zone electrophoresis (CZE-UV, Beckman Coulter P/ACE System MDQ), laboratory 8; circular dichroism (CD, AVIV Biomedical $430 \mathrm{CD}$ spectrophotometer), laboratory 11; differential scanning calorimetry (DSC, MicroCal VP DSC spectrometer), laboratory 9; intrinsic protein fluorescence (IPF, Varian Cary Eclipse spectrophotometer), laboratory 9; melting point fluorescence ( $T_{\mathrm{m}}$, Unchained Laboratories Optim and UNit systems), laboratory 13; HDX-MS (PAL HDX automation robot with Synapt G2Si or G2S, see Table S2), laboratories 1-4 and 12; IMS-MS (Waters Synapt G1/G2/G2Si instruments), laboratories 1, 3, 5, and 6; Raman (Renishaw inVia confocal Raman microscope), laboratory 7; SEC-UV (Agilent 1000 series HPLC), laboratory 1 ; Nb2.11 cell-based bioassay (CellTiterGlo, Promega), laboratory 10.

Details in full for each analytical methodology, analysis, and any deviations from the WI for sample preparation can be found in the Supporting Information.

\section{RESULTS AND DISCUSSION}

Development of Reference Protocol. Characterization of Secondary Structure Changes by HDX-MS. The secondary structure of rhGH was initially determined by HDX-MS experiments. In order to define measurement variability and establish measurement thresholds that discriminate changes in structural dynamics from HDX-MS measurement variability, a vial-to-vial study of rhGH was carried out. The differential HDX-MS measurements from triplicate interday data sets were processed using the statistical approach described by Groves et al. ${ }^{14}$ In Table S8, the raw peptide level thresholds are displayed. Differential HDX-MS experiments were then carried out to compare the structural dynamic of rhGH in the absence and presence of zinc also by using a statistical approach as described by Groves et al. ${ }^{14}$

The differential sums (relative measurements summed across all incubation periods) for each peptide were calculated for the comparison of (a) rhGH control and rhGH:Zn 1:2 and (b) rhGH control and rhGH:Zn 1:10 (Figure S-1 and Table S8). Based on the calculated thresholds, ${ }^{14}$ two peptides were identified in part (a) and 13 peptides in part (b) as representative of significant changes in structural dynamics of rhGH induced by the presence of zinc (Table 1).

Table 1. rhGH Peptides Identified as Showing Significant Changes Due to the Presence of Zinc, by HDX-MS Differential Analysis ${ }^{a}$

\begin{tabular}{|c|c|c|c|c|c|}
\hline & ide & ifier & $\begin{array}{r}\text { relat } \\
\mathrm{c}\end{array}$ & $\begin{array}{l}\text { HDX } \\
\text { ge }\end{array}$ & \\
\hline peptide, i & peptide ID & sequence & $\begin{array}{l}\mathrm{Zn} \\
1: 2\end{array}$ & $\begin{array}{c}\mathrm{Zn} \\
1: 10\end{array}$ & $\begin{array}{l}\text { region } \\
\text { ID }\end{array}$ \\
\hline 1 & $1-9$ & FPTIPLSRL & & - & I \\
\hline 2 & $2-9$ & PTIPLSRL & & - & I \\
\hline 4 & $14-25$ & MLRAHRLHQLAF & & + & II \\
\hline 5 & $15-25$ & LRAHRLHQLAF & + & + & II \\
\hline 6 & $16-25$ & RAHRLHQLAF & & + & II \\
\hline 7 & $26-32$ & DTYQEFE & + & + & II \\
\hline 26 & $93-100$ & LRSVFANS & & + & III \\
\hline 34 & $114-124$ & LKDLEEGIQTL & & + & IV \\
\hline 35 & $115-124$ & KDLEEGIQTL & & + & IV \\
\hline 36 & $117-124$ & LEEGIQTL & & + & IV \\
\hline 51 & $178-191$ & RIVQCRSVEGSCGF & & - & $\mathrm{V}$ \\
\hline 52 & $182-191$ & CRSVEGSCGF & & - & $\mathrm{V}$ \\
\hline 53 & $183-191$ & RSVEGSCGF & & - & $\mathrm{V}$ \\
\hline
\end{tabular}

${ }^{a}$ Changes are expressed as HDX increases (+) and decreases (-) relative to rhGH control; no significant change is indicated by blank input.

For the rhGH:Zn 1:2 sample, the two relevant peptides demonstrating changes in dynamics are located in region II, which is helix A of rhGH (Figure 1 and Figure S-1), where two of the three residues reported to be involved in the $\mathrm{Zn}^{2+}$ binding to rhGH are also located (His18 and His21). ${ }^{5,10}$ Increasing the concentration of zinc to obtain a ratio $\mathrm{rhGH}: \mathrm{Zn}$ equal to $1: 10$ confirmed the structural differences identified for the rhGH:Zn 1:2. Furthermore decreased HDX kinetics were observed at the $\mathrm{N}$ - and $\mathrm{C}$-terminal regions (regions I and $\mathrm{V}$ ) of the polypeptide chain, suggesting long-range changes in structural dynamics (Figure 1 and Figure S-1).

Structural destabilization in the form of increased HDX kinetics was also observed for regions III and IV in the presence of zinc, corresponding to both a loop region and part of helix C, respectively. All regions identified as showing dynamic changes due to the presence of zinc appeared to exhibit EX2 kinetics in both the absence and presence of zinc suggesting stable folded conformations on these time scales.

Definition of the Protocol for Sample Preparation. To ensure that the experimental conditions for the preparation of rhGH were adequate for the interlaboratory comparison and for the operative conditions of the diverse analytical platforms, additional experiments were carried out by SEC, IMS-MS, and IPF (using Optim platform). To ensure that dialysis had no significant effect on protein HOS, the thermal stability of 


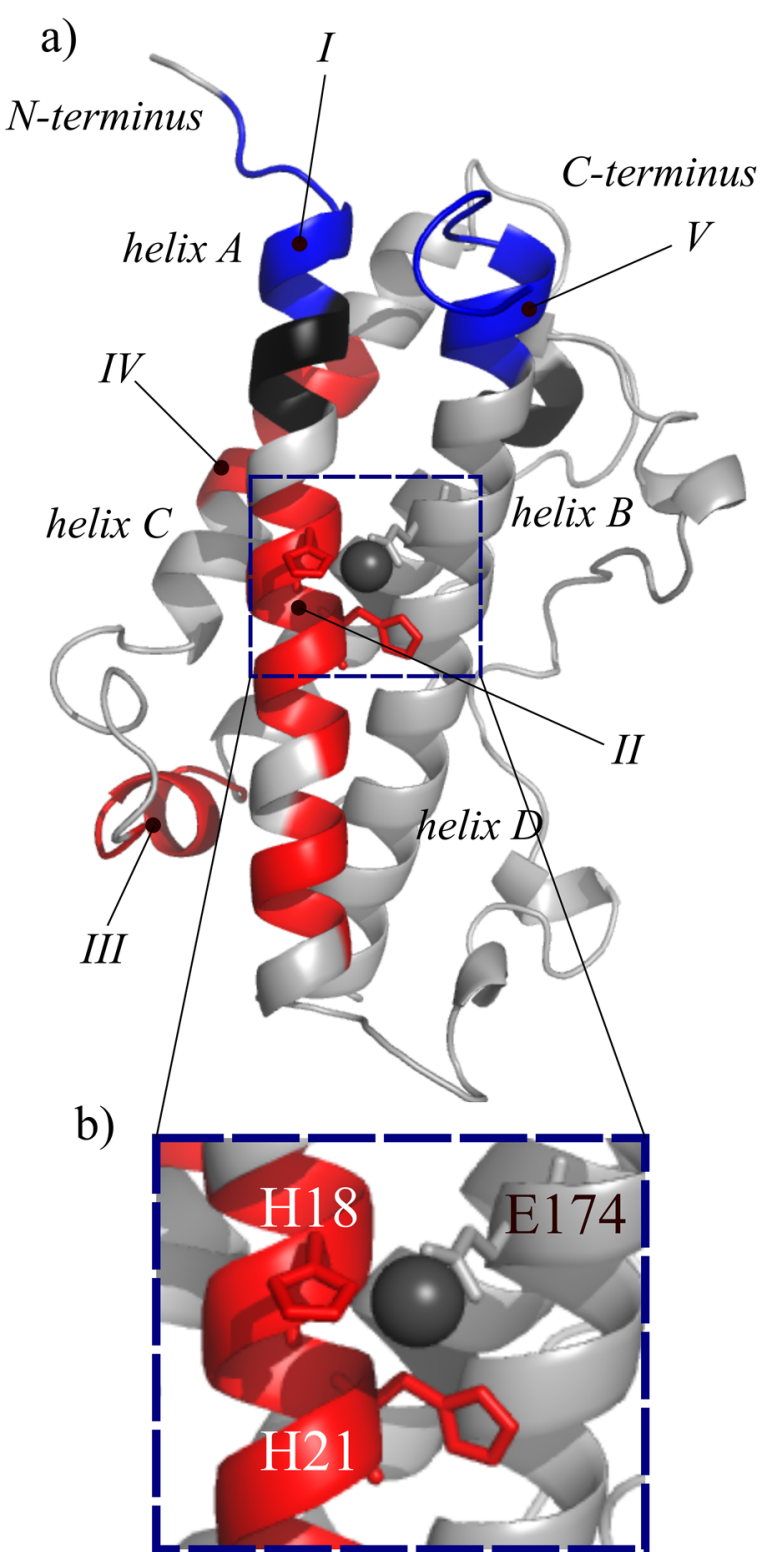

Figure 1. Relative differences in HDX-MS measurements between rhGH control and rhGH:Zn 1:10 samples. (a) Increased (red), decreased (blue), and unchanged (gray) HDX rates are indicated on the XRC structure of $\mathrm{rhGH}\left(\mathrm{PDB} 1 \mathrm{BP} 3^{5}\right)$. Missing peptidic sequences are indicated in black. A bound zinc ion is indicated as a sphere. Labeled regions include the following residues: region I [19], II [14-32], III [98-106], IV [114-124], V [178-191]. (b) Zinc binding residues His18, His21, and Glu174 are illustrated by stick representations. Measurements were provided by laboratory 1 , and relative changes were deemed significant using thresholds established using a vial-to-vial approach. ${ }^{14}$

rhGH samples was measured using IPF in both dialyzed and nondialyzed forms. Both samples exhibited the same change in fluorescence intensity (FLI) over temperature profiles, with a transition starting around $75{ }^{\circ} \mathrm{C}$ and a melting temperature $\left(T_{\mathrm{m}}\right)$ of $78.1 \pm 0.001{ }^{\circ} \mathrm{C}$ (Figure $\mathrm{S}-2$ ), suggesting consistent structures.

SEC-UV measurements were performed at two different concentrations of rhGH control $(7.2$ and $36 \mu \mathrm{M})$ covering the range of concentrations required for the diverse analytical experiments to monitor for the presence of aggregates. A peak eluting prior to the rhGH monomeric peak $(\sim 22 \mathrm{kDa})$ and attributed to rhGH dimers $(\sim 44 \mathrm{kDa})$ was consistently measured as $\leq 0.5 \%$ of rhGH signal across all samples (Figure S-3).

Native IMS-MS experiments were also carried out to monitor for the formation of potential hGH dimers induced by an increasing amount of zinc. Also in this case, the dimer population observed was consistently less than $0.5 \%$ (Figure S4).

Finally, the solubility of zinc acetate in KPBS was verified by measuring the content of soluble zinc by inductively coupled plasma optical emission spectrometry (Supporting Information). The measured content of zinc in the vials was within the expected measurement uncertainty.

$\mathrm{Zn}^{2+}$-rhGH binding was confirmed by native IMS-MS measurements (Figure S-4). Stochastic binding ratios of $1: 1$, and both $1: 1$ and $1: 2$ were observed for rhGH:Zn 1:2 and 1:10 samples, respectively. A control sample containing rhGH in the presence of ten molar equivalents of $\mathrm{Mg}^{2+}$ ( $\mathrm{rhGH}: \mathrm{Mg}$ 1:10) was also analyzed by native IMS-MS. The results showed reduced binding of the $\mathrm{Mg}^{2+}$ compared to $\mathrm{Zn}^{2+}$, confirming the specific binding of zinc (Figure S-4).

Interlaboratory Comparison Results. HDX-MS. Bottom up HDX-MS analysis was performed by five laboratories, with little guidance provided with respect to the experimental parameters (only a suggested incubation range) as for the other techniques used in this study. This was to define the potential utility of the rhGH:Zn protocol to aid method development and to assess instrument performance in different conditions and for diverse purposes.

Peptide maps from HDX-MS measurements submitted by the five laboratories varied in terms of the number of peptides (35-68), sequence coverage (82.2-96.9\%), and redundancy of overlapping peptidic sequences (2.20-4.20) as different methods were used. ${ }^{14,15}$ However, the sequence coverage across all data sets allowed for monitoring of most of the rhGH structure (Table S9 for individual laboratory sequence coverage data and Tables $\mathrm{S} 1-\mathrm{S} 5$ for experimental conditions), and comparable changes in the structural dynamics were observed particularly for the rhGH:Zn 1:10 sample.

The HDX-MS data from the different laboratories were also submitted as raw data and processed by the coordinating laboratory for comparison. For this, two tools widely used by the scientific community were applied, the commercially available DynamX software (based on the $3 \sigma$ approach) and the freely available online MEMHDX software. ${ }^{16}$ The HDXMS data from laboratory 1 , which were used to establish the protocol, were also reprocessed alongside the other data sets. Details on the parameters used for the statistical approaches are described in the Supporting Information. Regions identified as showing significant changes in dynamics using interpretation by DynamX or MEMHDX are indicated in Figure 2 and Figures S-5 and S-6. At higher concentrations of zinc, changes in the protein dynamics were observed by all laboratories as shown in Figure 2 and Figure S-5. Furthermore, by using the MEMHDX approach, subtle changes in kinetics $(\sim \leq 5 \%)$ were observed for the rhGH:Zn $1: 2$ by all laboratories and a decreased HDX kinetics at the N-terminal region of rhGH was also identified (Figure S-6).

Overall the results from laboratories $1-4$ processed by DynamX and MEMHDX also agreed with the results from laboratory 1 processed by applying the statistical approach described by Groves at al. ${ }^{14}$ 

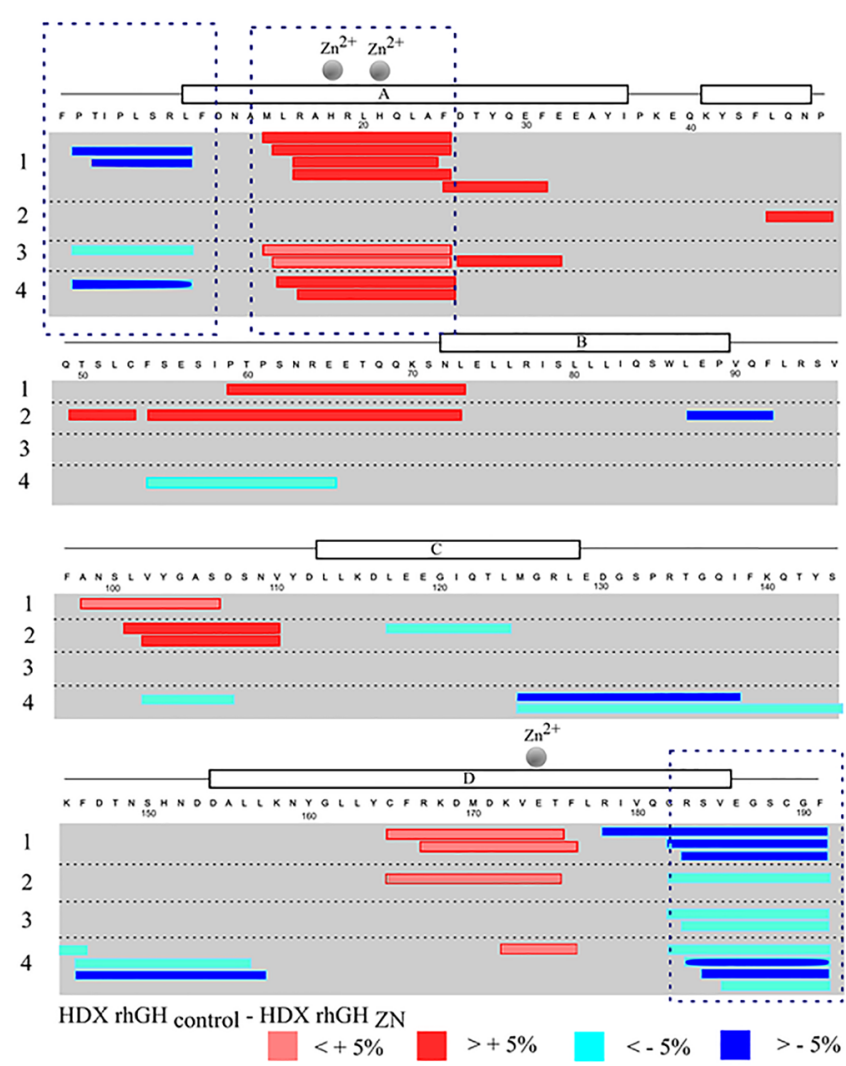

Figure 2. Relative deuterium uptake differences obtained by laboratories $1-4$ as indicated when considering differential HDXMS measurements between the rhGH control and rhGH:Zn 1:10 samples. Relative changes were deemed significant using the MEMHDX software tool. Increased (red), decreased (blue), and unchanged (gray) HDX rates of specific peptides are indicated on the sequence of rhGH with highlighted helices A, B, C, and D. Regions which are indicated as showing consistent structural changes across $\geq 3$ laboratories are highlighted in boxes. The spheres indicate zinc binding residues.

One laboratory performed HDX-MS measurements using incubation periods in the $\mathrm{ms}^{-1}-\mathrm{s}^{-1}$ range (Table S1, Figures S5 and S9) by using the setup described by Kish et al. ${ }^{18}$ This is an experimental approach which facilitates insights into more flexible/unstructured regions of a protein that fully deuterates using a $\geq$ millisecond time scale. ${ }^{17-19}$ Also in this case, structural differences were observed in the presence of zinc (Figure S-5). These data were consistent with the $\mathrm{Zn}$ dependent slower kinetics (same sign and similar magnitude) observed for $\mathrm{N}$ - and C-terminal regions identified across the other data sets. Although, in some cases, spectral envelopes indicate kinetics belonging to a mixed protein population were observed, there was a major species with EX2 behavior (Figure S-7), therefore deuterium uptake differences were calculated with the same method as for other data (delta centroid). Furthermore, as expected, the increased kinetics observed for the central region of helix $\mathrm{A}$ in the presence of zinc, only observed at later incubation periods during the conventional $>\mathrm{s}^{-1}$ HDX experimental setup were not observed using these shorter incubation periods. Further experiments outside the scope of this paper should be carried out both to confirm this and to determine if millisecond-mixing introduced a mixing artifact or if there is evidence of a meaningful mixed protein population under HDX labeling conditions (e.g., monomer/ dimer).

Native IMS-MS. Native IMS-MS measurement revealed three different conformations of rhGH: a compacted monomer, an extended monomer, and a dimeric species, as reported for all rhGH and rhGH:Zn samples by all four participating laboratories. Agreement between laboratories was also observed for stoichiometries of $1: 1$ and $1: 2 \mathrm{rhGH}: \mathrm{Zn}$ with an increased propensity of the 1:2 binding ratio for the rhGH:Zn 1:10 sample.

Reported collisional cross section $\left({ }^{\mathrm{TW}} \mathrm{CCS}_{\mathrm{N} 2}\right)$ measurements for the +9 charge state of $\mathrm{rhGH}$ and $\mathrm{rhGH}: \mathrm{Zn}$ samples, as well as instrumentation used, are summarized in Table 2. All

Table 2. Reported ${ }^{\mathrm{TW}} \mathrm{CCS}_{\mathrm{N} 2}$ Measurement Values for the +9 Charge State of rhGH Control and rhGH:Zn Bound Species $^{a}$

\begin{tabular}{|c|c|c|c|c|}
\hline $\begin{array}{l}\text { lab } \\
\text { ID }\end{array}$ & instrument & $\operatorname{rhGH}\left(\AA^{2}\right)$ & $\begin{array}{c}\mathrm{rhGH}: \mathrm{Zn} 1: 2 \\
\left(\AA^{2}\right)\end{array}$ & $\underset{\left(\AA^{2}\right)}{\mathrm{rhGH}: \mathrm{Zn} 1: 10}$ \\
\hline 1 & Synapt G2Si & $2482 \pm 162$ & $2480.9 \pm 161$ & $2486.6 \pm 162$ \\
\hline 3 & Synapt G2Si & $2322.2 \pm 19$ & $2264.5 \pm 6$ & $2269.0 \pm 11$ \\
\hline 5 & $\begin{array}{l}\text { Synapt } \\
\text { HDMS }\end{array}$ & $2129 \pm 48$ & $2135 \pm 50$ & $2106 \pm 52$ \\
\hline 6 & Synapt G2 & $2103 \pm 12$ & $2088 \pm 9$ & $2019 \pm 2$ \\
\hline
\end{tabular}

${ }^{a}$ All measurements were performed using Synapt Tri-Wave based IMS instruments of varying generations as stated. Uncertainty values stated were generated by the participating laboratory.

measurements were performed using the Synapt Tri-Wave instrument series [Waters, Milford] of varying generations. An apparent correlation between the IMS-MS instrument generation and reported ${ }^{\mathrm{TW}} \mathrm{CCS}_{\mathrm{N} 2}$ measurements of the rhGH control appears to exist, with measurements in the range $\sim 2100$ and $\sim 2400 \AA^{2}$ for earlier generation (HDMS/ G2) and G2Si Synapt instrumentation, respectively, suggesting instrumental bias on measurements. ${ }^{\mathrm{TW}} \mathrm{CCS}_{\mathrm{N} 2}$ measurements of rhGH using a HDMS instrument are consistent with previously published ${ }^{\mathrm{TW}} \mathrm{CCS}_{\mathrm{N} 2}$ measurements of $2077 \pm 230$ $\AA^{2}{ }^{20}$ Though changes in ${ }^{\mathrm{TW}} \mathrm{CCS}_{\mathrm{N} 2}$ measurements between the rhGH and rhGH-Zn bound species were only reported by two laboratories, measurements from all laboratories demonstrated narrower full width half-maximum (fwhm) for arrival time distribution of IMS-MS measurements of the $\geq+9$ charge state in rhGH:Zn 1:10 samples for singly rhGH-Zn bound species, relative to rhGH control and rhGH:Zn 1:2 samples (Figure $S$ $8)$. These data suggest the distribution of species present is more stable and compact when zinc is bound.

Two laboratories ( 1 and 5) also performed collisional activation (CA)-IMS-MS experiments carried out on free and zinc bound charge species of $\mathrm{rhGH}$, whereby both data sets revealed an increase in stability $\sim 2 \mathrm{~V}$ against unfolding for the zinc bound species relative to the free species, Figure S-9. Overall an apparent global stabilization of rhGH in the presence of zinc was observed with no large global structural rearrangement.

Raman Spectroscopy. Following dialysis and dried spot preparation, Raman spectra were recorded for all rhGH and rhGH:Zn samples. Analysis of measurements of the rhGH control and rhGH:Zn 1:2 and 1:10 samples allowed subtle differences between the samples to be observed at some specific wavenumbers. Suggested peak assignments have been made for these regions of changes in shift intensity (Figure 3, Table S10). In particular, small changes in peak intensity were 


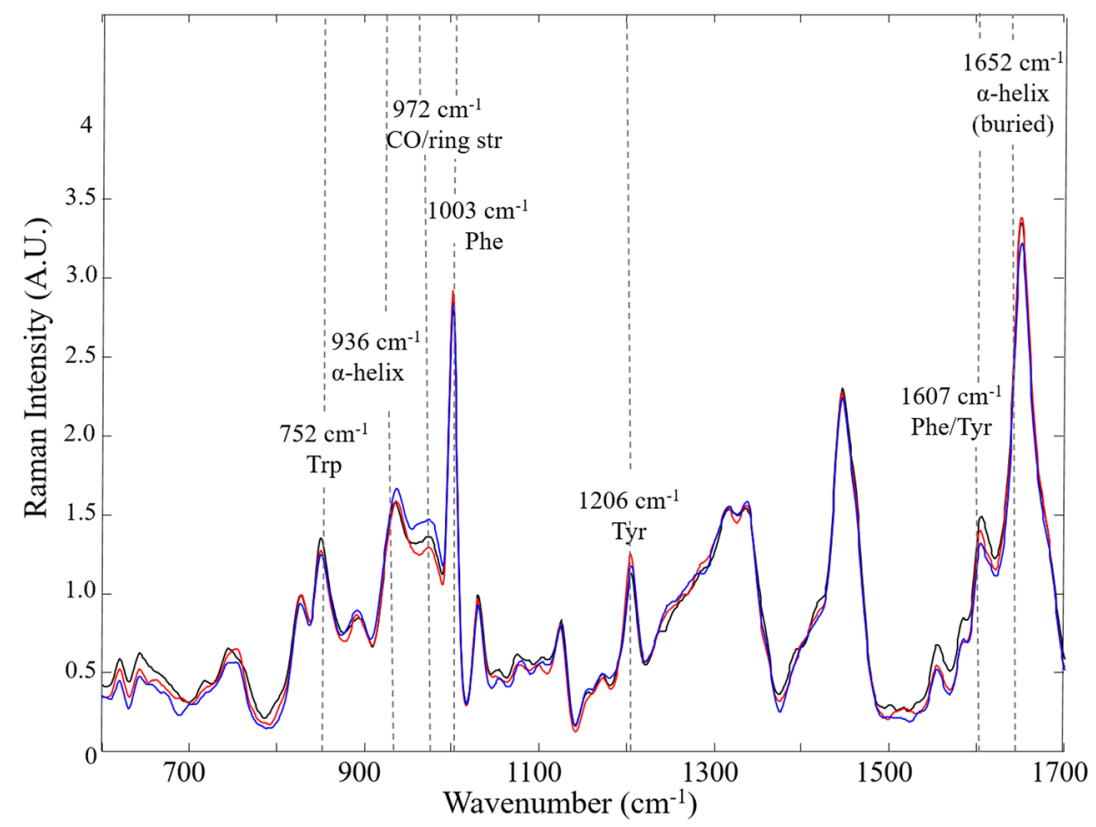

Figure 3. Averaged Raman spectra $(n=6)$ of dialyzed rhGH samples, rhGH control (black), rhGH:Zn 1:2 (red), and rhGH:Zn 1:10 (blue) after preprocessing (normalized, baselined, and smoothed). Selected wavenumbers showing intensity shifts across samples are indicated.

Table 3. Summary of Melting Temperatures $\left(T_{\mathrm{m}}\right)$ Measured for rhGH Control, rhGH:Zn 1:2, and rhGH:Zn 1:10 for Two Different Analytical Platforms ${ }^{a}$

\begin{tabular}{cccr} 
& & & \\
& & & \\
\cline { 3 - 4 } platform & rhGH concentration & rhGH control & rhGH:Zn $1: 2$ \\
UNit & $36 \mu \mathrm{M}$ & $81.20 \pm 0.25$ & $82.00 \pm 0.50$ \\
DSC & $25 \mu \mathrm{M}$ & $78.79 \pm 0.06$ & $79.08 \pm 0.15$
\end{tabular}

${ }^{a}$ Sample concentration and platform are indicated; method details can be found in the Supporting Information.

observed at 1206,1607 , and $1652 \mathrm{~cm}^{-1}$ for the rhGH:Zn $1: 10$ sample compared to the rhGH control and rhGH:Zn 1:2 samples, associated with the loss of a buried $\alpha$-helical structure and changes in orientation of tyrosine and phenylalanine side chain residues. $^{21-23}$ These spectral changes, although subtle, potentially correspond to a change in conformation for a buried $\alpha$-helical structure which would be consistent with the zinc binding site located at helices $\mathrm{A}$ and $\mathrm{D}$ and observations of HDX-MS measurements.

$C D$. CD measurements of $36 \mu \mathrm{M}$ rhGH samples were shown to be reproducible across all samples and replicates (Figure S10). The relative percentage content of various secondary structural features as determined from the $\mathrm{CD}$ data are as follows ( $\alpha$-helical, $\beta$-sheet, turns, unstructured): rhGH control (61\%, 3\%, 14\%, 22\%), rhGH:Zn 1:2 (60\%, 3\%, 14\%, 23\%), and rhGH:Zn 1:10 (57\%, 3\%, 16\%, 24\%); hence, all samples were predominantly $\alpha$-helical in structure. Secondary structures of the rhGH control and rhGH:Zn 1:2 samples were equivalent; however, for the rhGH:Zn 1:10 samples, the $\alpha$ helix content was lower by $4 \%$, with concomitant increases of $2 \%$ in both turn and unstructured types, indicating a modest but significant structural change at this concentration of zinc. These data are consistent with HDX-MS and Raman measurements which demonstrate a loss of the $\alpha$-helical structure upon zinc binding.

Capillary Zone Electrophoresis. Analysis by CZE-UV revealed two peaks in the CZE profiles of each rhGH and rhGH:Zn samples, one peak attributed to excipients and the other to the monomeric rhGH (Figure S-11), as demonstrated by ultrafiltration experiments and consequent injection of the retained and filtrated samples in CZE (data not shown, see the Supporting Information). Reproducible electrophoretic mobilities were observed across all rhGH and rhGH:Zn samples, suggesting consistent globular structures across the samples.

Intrinsic Fluorescence. Tertiary structural changes between the rhGH and rhGH:Zn 1:10 samples were monitored using intrinsic tryptophan fluorescence whereby no shift in $\lambda$ max was observed across the rhGH samples (Table S11) suggesting no sensitivity of the technique to tertiary structural changes upon $\mathrm{Zn}^{2+}$ binding.

Thermal Stability. Two different analytical platforms, UNit and DSC, were used to assess the thermal stability of rhGH and rhGH:Zn samples, measurements are summarized in Table 3. Overall high measurement comparability was shown by the two thermal stability platforms for the rhGH:Zn $1: 10$ samples, a consistent and reproducible increase in $T_{\mathrm{m}}$ of $\sim 1{ }^{\circ} \mathrm{C}$ for rhGH:Zn 1:10 samples compared to the rhGH control, at 25 and $36 \mu \mathrm{M}$ concentrations, was reported for both platforms. A smaller $\left(\sim 0.3{ }^{\circ} \mathrm{C}\right)$ but reproducible increase in $T_{\mathrm{m}}$ for rhGH:Zn 1:2 samples compared to the rhGH control was measured by DSC only (Figure S-12). These data suggest an increase in global stability in the presence of zinc, with the magnitude of global stabilization increasing at higher zinc concentrations. No evidence of temperature induced aggregation was observed for the samples at either concentration.

Analytical Ultracentrifugation (AUC). AUC analysis measured the Stokes radii of the rhGH control sample to be $2.37 \mathrm{~nm}$, with a decreasing Stokes radii observed upon the 
Table 4. Summary of the Sensitivity of the Range of Analytical Platforms and Methods Across the Interlaboratory Comparison Towards Relative Structural Changes between rhGH Control Sample and rhGH:Zn Samples at Ratios of 1:2, 1:5, and 1:10 ${ }^{a}$

\begin{tabular}{|c|c|c|c|c|c|c|c|}
\hline Analytical platform & $\begin{array}{l}\text { Lab } \\
\text { ID }\end{array}$ & $\begin{array}{c}\text { rhGH:Zn } \\
1: 2\end{array}$ & $\begin{array}{c}\text { rhGH:Zn } \\
1: 5\end{array}$ & $\begin{array}{c}\text { rhGH:Zn } \\
1: 10\end{array}$ & Structural information type & $\begin{array}{c}\text { Sample } \\
\text { concentration }\end{array}$ & $\begin{array}{l}\text { Dialysis } \\
\text { required }\end{array}$ \\
\hline \multirow{4}{*}{ HDX-MS * } & 1 & & & & \multirow{5}{*}{ localised secondary } & \multirow{5}{*}{$7.2 \mu \mathrm{M}$} & \multirow{5}{*}{$\mathrm{N}$} \\
\hline & 2 & & & & & & \\
\hline & 3 & & & & & & \\
\hline & 4 & & & & & & \\
\hline msHDX-MS * & 12 & & & & & & \\
\hline \multirow{2}{*}{$\begin{array}{l}\text { IMS-MS } \\
\text { (inc. CIA-IMS-MS) }\end{array}$} & 1 & & & & \multirow{4}{*}{$\begin{array}{l}\text { global tertiary, quaternary, charge and stability } \\
\text { (CIA-IMS-MS) }\end{array}$} & \multirow{4}{*}{$7.2 \mu \mathrm{M}$} & \multirow{4}{*}{ Y } \\
\hline & 5 & & & & & & \\
\hline \multirow{2}{*}{ IMS-MS } & 3 & & & & & & \\
\hline & 6 & & & & & & \\
\hline SEC-UV & 1 & & & & global tertiary and quaternary & $7.2-45 \mu \mathrm{M}$ & $\mathrm{N}$ \\
\hline Raman spectroscopy & 7 & & & & Secondary & Dried $\sim \mathrm{nM}$ & $\mathrm{Y}$ \\
\hline CZE & 8 & & & & global tertiary and quaternary and charge & $7.2 \mu \mathrm{M}$ & $\mathrm{N}$ \\
\hline IPF & 9 & & & & global tertiary & $36 \mu \mathrm{M}$ & $\mathrm{Y}$ \\
\hline DSC & 9 & & & & \multirow{2}{*}{ global tertiary and stability } & $25 \mu \mathrm{M}$ & $\mathrm{Y}$ \\
\hline UNit & 13 & & & & & $36 \mu \mathrm{M}$ & $\mathrm{N}$ \\
\hline AUC & 9 & & & & global tertiary & $22.6 \mu \mathrm{M}$ & $\mathrm{Y}$ \\
\hline Bio-assay & 10 & & & & biological activity & $\begin{array}{c}0.35-0.45 \\
\mathrm{nM}\end{array}$ & $\mathrm{N}$ \\
\hline $\mathrm{CD}$ & 11 & & & & secondary structure & $36 \mu \mathrm{M}$ & $\mathrm{N}$ \\
\hline
\end{tabular}

${ }^{a}$ Positive (green), negative (red), and nonapplicable (blank) identification of a structural change are indicated, as are Lab ID, sample concentration, the type of structural information gained during the analyses and if dialysis of the sample was performed prior to analysis ( $\mathrm{Y}$, yes or $\mathrm{N}$, no). Structural changes as indicated with an * using MEM-HDX data analysis workflows to assess data significance.

addition of zinc to $\mathrm{rhGH}$, of $\sim 1.8$ and $7.1 \%$ for rhGH:Zn ratios of $1: 2$ and 1:10, respectively (Table S12, Figure S-13), suggesting the global structure of rhGH has become more spherical/compact in increasing presence of zinc. Other than a change in conformation, a shift in the distribution of monomer/dimer populations could account for this change in Stokes radii. However, this was ruled out as no evidence of aggregation was observed with the addition of zinc, upon visual inspection of the sample nor by SEC-UV or thermal denaturation measurements.

Nb2.11 Cell-Based Bioassay. Assay measurements were performed across the $0.35-45 \mathrm{nM}$ range using two $\mathrm{Nb} 2.11$ cell-based bioassays. No change in activity was observed for rhGH preincubated in the presence zinc at $\mathrm{rhGH}: \mathrm{Zn}$ ratios of $1: 2$ and 1:5 relative to the rhGH control. While no change in activity was observed across the samples, it has to be considered that the extensive dilution of the samples and the need to perform the bioassay in a complex assay medium (including Fishers medium and $1 \% \mathrm{v} / \mathrm{v} /$ heat-inactivated horse's serum) may have altered the availability of the zinc ions. A secondary assay measuring the proliferative response of $\mathrm{Nb} 2.11$ cells upon the direct addition of zinc acetate, saw a reduced cellular response. However, this was deemed insignificant due to the evidence of increased cell death in negative controls containing zinc acetate only. Additionally it is possible that the changes in HOS may have no impact on the activity of rhGH in the assay.

Comparability of Results. Table 4 summarizes the results from the interlaboratory comparison and the suitability of the protocol to assess the sensitivity to structural changes and structural dynamics of diverse analytical platforms. Particularly HDX-MS, IMS-MS, DSC, and AUC measurements showed structural changes of rhGH at both concentrations of zinc. In contrast, measurements performed by more routine analytical platforms such as the SEC-UV, CZE, and IPF measurements were unable to detect any structural changes using this model system. Induced changes in structural dynamics were reproducibly resolved to specific regions of the rhGH structure by HDX-MS measurements performed in multiple laboratories, despite the nonstandardized conditions. A particular correlation was seen across HDX-MS, CD, and Raman spectroscopic measurements and provided complementary information. A loss in helical structure upon the addition of zinc was observed, suggesting localized structural changes induced by the protocol, though HOS changes remain unresolved to regions for CD and Raman data sets. Similarly, native IMS-MS, thermal stability, and AUC measurements indicated a small and consistent globular structural change, though with varying sensitivity.

\section{CONCLUSIONS}

A protocol to assess sensitivity to protein HOS changes and validate analytical platforms used for measuring those changes was here successfully developed and its validity demonstrated across a number of analytical platforms. To our knowledge, this is the first protocol and interplatform study of its kind that enable a comparability investigation of HOS measurements across a plethora of methodologies, including established and emerging technologies, spanning the breadth of the biopharmaceutical toolbox for HOS measurements. The range of resolution and sensitivity observed across platforms illustrates the struggles of the biopharmaceutical industry when met with the task of providing sufficient evidence to regulators for the continuity of protein structure during both $\mathrm{R} \& \mathrm{D}$ and manufacturing stages of drug development. Rather these data demonstrate the need for multiple sources of measurements to be used in conjunction with one another. The availability of suitable quality control materials to provide such evidence to regulators and industry will be a key component in its more widespread adoption (alongside easier/more accessible data analysis) within this setting. Undoubtable similar protocols and approaches should be developed for a broader range of 
bioproducts, including monoclonal antibodies. Those studies will provide complementary tools and information to the one generated from standardized interlaboratory comparisons to assess the reproducibility of methods such as the HDX-MS interlaboratory comparison described in Hugdens et al. ${ }^{24}$

\section{ASSOCIATED CONTENT}

\section{SI Supporting Information}

The Supporting Information is available free of charge at https://pubs.acs.org/doi/10.1021/acs.analchem.0c04625.

Additional experimental details including sample preparations, all analytical methodologies, experimental measurements both in graphical and tabulated formats (ICP-EOS, HDX-MS, IPF, SEC-UV, native IMS-MS, CA-IMS-MS, CD, CZE-UV, AUC, Raman, DCS) (PDF)

Generic working instructions for higher order protein structural analysis comparison for the interlaboratory comparison (PDF)

HDX-MS working instructions for higher order protein structural analysis comparison for the interlaboratory comparison (PDF)

IMS-MS working instructions for higher order protein structural analysis comparison for the interlaboratory comparison (PDF)

Summary of the raw data from HDX-MS experiments (XLSX)

\section{AUTHOR INFORMATION}

\section{Corresponding Authors}

M. Quaglia - National Measurement Laboratory, LGC Ltd., Teddington, Middlesex TW11 OLY, U.K.;

Email: milena.quaglia@lgcgroup.com

K. Groves - National Measurement Laboratory, LGC Ltd.,

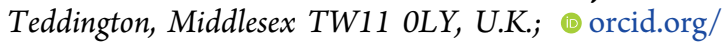
0000-0001-9207-6392; Email: katejgroves@gmail.com

\section{Authors}

A. E. Ashcroft - Astbury Centre for Structural Molecular Biology \& School of Molecular and Cellular Biology, University of Leeds, Leeds LS2 9JT, U.K.; (1) orcid.org/ 0000-0002-1676-123X

A. Cryar - National Measurement Laboratory, LGC Ltd., Teddington, Middlesex TW11 OLY, U.K.

A. Sula - Institute of Structural and Molecular Biology, Birkbeck College, University of London, London WC1E 7HX, U.K.

B. A. Wallace - Institute of Structural and Molecular Biology, Birkbeck College, University of London, London WC1E 7HX, U.K.

B. B. Stocks - National Research Council Canada, Ottawa K1A 0R6, Canada; 이이이.org/0000-0002-7265-9344

C. Burns - Biotherapeutics Division, National Institute for Biological Standards and Control, Blanche Lane South Mimms, Potters Bar, Hertfordshire EN6 3QG, U.K.

D. Cooper-Shepherd - National Measurement Laboratory, LGC Ltd., Teddington, Middlesex TW11 OLY, U.K.

E. De Lorenzi - Department of Drug Sciences, University of Pavia, 27100 Pavia, Italy; 10 orcid.org/0000-0002-54507208

E. Rodriguez - UCB Celltech, Slough, Berkshire SL1 3WE, U.K.
H. Zhang - Department of Biochemical Engineering, University College London, London WC1E 6BT, U.K.

J. R. Ault - Astbury Centre for Structural Molecular Biology \& School of Molecular and Cellular Biology, University of Leeds, Leeds LS2 9JT, U.K.

J. Ferguson - Biotherapeutics Division, National Institute for Biological Standards and Control, Blanche Lane South Mimms, Potters Bar, Hertfordshire EN6 3QG, U.K.

J. J. Phillips - Living Systems Institute, Department of Biosciences, University of Exeter, Exeter EX4 4QD, U.K.; (1) orcid.org/0000-0002-5361-9582

K. Pacholarz - Manchester Institute of Biotechnology, The University of Manchester, Manchester M1 7DN, U.K.

K. Thalassinos - Institute of Structural and Molecular Biology, Division of Biosciences, University College London, London WC1E 6AR, U.K.; 1 orcid.org/0000-0001-50728428

L. Luckau - National Measurement Laboratory, LGC Ltd., Teddington, Middlesex TW11 OLY, U.K.

L. Ashton - Department of Chemistry, Lancaster University, Lancaster LA1 4YB, U.K.; (1) orcid.org/0000-0002-13236309

O. Durrant - UCB Celltech, Slough, Berkshire SL1 3WE, U.K.

P. Barran - Manchester Institute of Biotechnology, The University of Manchester, Manchester M1 7DN, U.K.; (1) orcid.org/0000-0002-7720-586X

P. Dalby - Department of Biochemical Engineering, University College London, London WC1E 6BT, U.K.; $\odot$ orcid.org/ 0000-0002-0980-8167

P. Vicedo - Manchester Institute of Biotechnology, The University of Manchester, Manchester M1 7DN, U.K.

R. Colombo - Department of Drug Sciences, University of Pavia, 27100 Pavia, Italy

R. Davis - UCB Celltech, Slough, Berkshire SL1 3WE, U.K.

R. Parakra - Living Systems Institute, Department of Biosciences, University of Exeter, Exeter EX4 4QD, U.K.

R. Upton - Manchester Institute of Biotechnology, The University of Manchester, Manchester M1 7DN, U.K.

S. Hill - National Measurement Laboratory, LGC Ltd., Teddington, Middlesex TW11 OLY, U.K.; ○ orcid.org/ 0000-0002-7480-0655

V. Wood - Department of Biochemical Engineering, University College London, London WC1E 6BT, U.K.

Z. Soloviev - Institute of Structural and Molecular Biology, Division of Biosciences, University College London, London WC1E 6AR, U.K.

Complete contact information is available at:

https://pubs.acs.org/10.1021/acs.analchem.0c04625

\section{Author Contributions}

The manuscript was written through contributions of all authors. All authors have given approval to the final version of the manuscript.

Notes

The authors declare no competing financial interest.

Data Availability: The circular dichroism spectra and meta-data have been deposited in the Protein Circular Dichroism Data Bank (PCDDB) located at http://pcddb.cryst.bbk.ac.uk (ID codes CD0006358000, CD0006362000, and CD0006363000). 


\section{ACKNOWLEDGMENTS}

The development of the protocol, coordination of the interlaboratory study, and collation of results described in this manuscript was funded by the U.K. government, Department for Business, Energy \& Industrial Strategy (BEIS). This work was supported, in part, by Grant BB/ P024092 from the U.K. Biotechnology and Biological Sciences Research Council to B. A. Wallace. The authors would also like to acknowledge Dr. Sebastien Brier for his assistance with MEMHDX analysis. Participants in the other aspects of this interlaboratory study kindly donated their analyses and time without funding contributions.

\section{REFERENCES}

(1) Urquhart, L. Nat. Rev. Drug Discovery 2020, 19 (2), 86-86.

(2) European Medicines Agency. ICH Q8R2 Guideline on Pharmaceutical Development, January 11, 2005.

(3) European Medicines Agency. ICH Q6B Specifications: Test Procedures and Acceptance Criteria for Biotechnological/Biological Products, October 3, 1999.

(4) European Medicines Agency. ICH Guideline Q11 on Development and Manufacture of Drug Substances (Chemical Entities and Biotechnological/Biological Entities), Step 3, May 2011.

(5) Somers, W.; Ultsch, M.; De Vos, A. M.; Kossiakoff, A. A. Nature 1994, 372 (6505), 478-481.

(6) Kasimova, M. R.; Kristensen, S. M.; Howe, P. W. A.; Christensen, T.; Matthiesen, F.; Petersen, J.; Sørensen, H. H.; Led, J. J. J. Mol. Biol. 2002, 318 (3), 679-695.

(7) Seger, S. T.; Breinholt, J.; Faber, J. H.; Andersen, M. D.; Wiberg, C.; Schjødt, C. B.; Rand, K. D. Anal. Chem. 2015, 87 (12), 59735980.

(8) WHO International Standard Somatropin (Recombinant DNADerived Human Growth Hormone); 98/574; MHRA, NIBSC, 2018; p 3.

(9) de Vos, A. M.; Ultsch, M.; Kossiakoff, A. A. Science 1992, 255

(5042), 306-312.

(10) Cunningham, B. C.; Bass, S.; Fuh, G.; Wells, J. A. Science 1990, 250 (4988), 1709-1712.

(11) Cunningham, B. C.; Mulkerrin, M. G.; Wells, J. A. Science 1991, 253 (5019), 545-548.

(12) Greenan, J.; Lorenson, M. Y.; Conconi, M. V.; Walker, A. M. Endocrinology 1990, 126 (1), 512-518.

(13) Yang, T. H.; Cleland, J. L.; Lam, X.; Meyer, J. D.; Jones, L. S.; Randolph, T. W.; Manning, M. C.; Carpenter, J. F. J. Pharm. Sci. 2000, 89 (11), 1480-1485.

(14) Groves, K.; Cryar, A.; Cowen, S.; Ashcroft, A. E.; Quaglia, M. J. Am. Soc. Mass Spectrom. 2020, 31, 553.

(15) Ahn, J. Local Hydrogen Deuterium Exchange Mass Spectrometry: From Pressurized Online Digestion to Pepsin Proteolysis. Ph.D. Thesis, Northeastern University, Boston, MA, 2013.

(16) Hourdel, V.; Volant, S.; O'Brien, D. P.; Chenal, A.; ChamotRooke, J.; Dillies, M.-A.; Brier, S. Bioinformatics 2016, 32 (22), 34133419.

(17) Keppel, T. R.; Weis, D. D. Anal. Chem. 2013, 85 (10), 51615168.

(18) Kish, M.; Smith, V.; Subramanian, S.; Vollmer, F.; Lethbridge, N.; Cole, L.; Bond, N.; Phillips, J. J. bioRxiv 2019, 654665.

(19) Rist, W.; Rodriguez, F.; Jørgensen, T. J. D.; Mayer, M. P. Protein Sci. 2005, 14 (3), 626-632.

(20) Pritchard, C.; O’Connor, G.; Ashcroft, A. Anal. Chem. 2013, 85, $7205-7212$.

(21) Takekiyo, T.; Imai, T.; Kato, M.; Taniguchi, Y. Biochim. Biophys. Acta, Proteins Proteomics 2006, 1764 (3), 355-363.

(22) Maiti, N. C.; Apetri, M. M.; Zagorski, M. G.; Carey, P. R.; Anderson, V. E. J. Am. Chem. Soc. 2004, 126 (8), 2399-2408.

(23) Hernández, B.; Tinacci, L.; Coïc, Y.-M.; Chenal, A.; Cohen, R.; Sanchez-Cortes, S.; Ghomi, M. J. Phys. Chem. C 2018, 122, 13034.
(24) Hudgens, J. W.; Gallagher, E. S.; Karageorgos, I.; Anderson, K. W.; Filliben, J. J.; Huang, R. Y.-C.; Chen, G.; Bou-Assaf, G. M.; Espada, A.; Chalmers, M. J.; Harguindey, E.; Zhang, H.-M.; Walters, B. T.; Zhang, J.; Venable, J.; Steckler, C.; Park, I.; Brock, A.; Lu, X.; Pandey, R.; Chandramohan, A.; Anand, G. S.; Nirudodhi, S. N.; Sperry, J. B.; Rouse, J. C.; Carroll, J. A.; Rand, K. D.; Leurs, U.; Weis, D. D.; Al-Naqshabandi, M. A.; Hageman, T. S.; Deredge, D.; Wintrode, P. L.; Papanastasiou, M.; Lambris, J. D.; Li, S.; Urata, S. Anal. Chem. 2019, 91 (11), 7336-7345. 